\title{
Ferramenta Computacional para Classificação de ECG Humanos com Detecção de Defeitos no Eletrocardiógrafo
}

\author{
Maycon E. F. Ribeiro \\ Lab. de Sistemas Distribuídos (LaSiD) \\ Dept. de Ciência da Computação (DCC) \\ Univ. Federal da Bahia (UFBA.) \\ Salvador, Bahia \\ mayconexa@hotmail.com
}

\author{
Juan A. L. Cruz \\ Lab. de Inst. em Física (LinFis) \\ Dept. de Física (DFIS.) \\ Univ. Est. de Feira de Santana (UEFS.) \\ Feira de Santana, Bahia \\ juan@uefs.br
}

\author{
Raimundo J. de A. Macêdo \\ Lab. de Sistemas Distribuídos (LaSiD) \\ Dept. de Ciência da Computação (DCC) \\ Univ. Federal da Bahia (UFBA.) \\ Salvador, Bahia \\ macedo@ufba.br
}

\begin{abstract}
Resumo-Há muito tempo, os eletrocardiogramas (ECGs) vêm sendo utilizados para diagnosticar problemas cardíacos. No entanto, obter uma classificação automática satisfatória de ECGs em sistemas de $e$-Health, é uma tarefa desafiadora, devido a interferências operacionais e falhas às quais esses dispositivos estão submetidos. Neste artigo, apresentamos a ferramenta computacional Cyber-ECG para classificação automática de sinais de ECG, com detecção de defeitos de sensores do eletrocardiógrafo. A Cyber-ECG foi implementada em ambiente Simulink/MATLAB e avaliada a partir de séries temporais de ECGs disponíveis em banco de dados público. A ferramenta proposta obteve uma precisão de $84 \%$ e $80 \%$ ao classificar arritmias e batimentos normais, respectivamente. Para essas mesmas classes, os valores de F1- Score são $82 \%$ e $83 \%$. Portanto, a ferramenta apresentou funcionamento satisfatório e, em alguns casos, teve desempenho superior em comparação com outros resultados de métodos de classificação de sinais de ECG relatados na literatura científica. O detector de defeito foi avaliado a partir de um módulo de injeção de falhas integrado à ferramenta Cyber-ECG, o que permitiu verificar a eficácia do método proposto.

Index Terms-Eletrocardiogramas, Classificação de ECG, $e$ -
\end{abstract} Health, Tolerância a Falhas.

\section{INTRODUÇÃO}

Conforme a Organização Mundial da Saúde (OMS), as doenças cardiovasculares são as principais causas de morte no mundo e se tornarão ainda mais prevalentes com o envelhecimento da população [1]. Em relatório de 2016, portanto antes da pandemia de COVID-19, a Sociedade Brasileira de Cardiologia reportou que as doenças associadas ao coração estavam entre as principais causas de morte no Brasil, provocando o falecimento de 350.000 pessoas por ano, no período considerado, o que representava $30 \%$ de todas as causas das mortes no país [2]. Além disto, estudos clínicos recentes relatam uma associação entre a doença do coronavírus 2019 (COVID-19) e as doenças cardiovasculares, podendo elevar consideravelmente esta estatística [3]. Portanto, não existem dúvidas de que as doenças do coração são um grave problema para a saúde humana em geral, e representam uns dos principais desafios das pesquisas contemporâneas na área biomédica.
Aprimorar ou criar tecnologias para melhorar o diagnóstico de doenças do coração em tempo real é crucial, porque, ao detectar a doença precocemente, existem mais possibilidades de cura e prevenção. Uma técnica que ganha cada vez mais destaque nesse cenário é o serviço de $e$-Health para Eletrocardiografia. Ou seja, eletrocardiogramas (ECGs) utilizando processos eletrônicos e de comunicação. Os ECGs representam o potencial elétrico medido em diferentes pontos da superfície do corpo, para avaliar o estado fisiológico do coração [4]. Estes sinais são séries temporais não-estacionárias e normalmente de baixa amplitude, o que torna difícil uma rápida verificação ou classificação precisa [5] e [6].

Uma dificuldade adicional para classificação de ECGs, decorre de inconsistências dos dispositivos usados para a aquisição dos sinais, quando estes sofrem defeitos no equipamento físico. Estes defeitos são causados, na sua maioria, pelo desgaste dos sensores, mau funcionamento dos equipamentos, problemas de conectividade dos sensores e, até mesmo, interferências provocadas pela radiação eletromagnética ao redor [7]. As principais consequências são a ausência ou mudanças bruscas nos padrões das ondas dos ECGs. Como não é possível controlar fatores que resultam em falhas, existe a necessidade de adicionar mecanismos auxiliares para melhorar a confiabilidade (reliability) no monitoramento do coração [8].

Para dar conta deste desafio, alguns trabalhos utilizam técnicas de Tolerância a Falhas (TF) para aumentar a robustez dos serviços de classificação de ECGs, de modo a evitar que situações de falhas comprometam os respectivos diagnósticos. Os estudos relacionados à TF para os serviços de $e$-Health, em sua maioria, recorrem a nós sensores redundantes (redundância de Hardware) com objetivo de mascarar falhas [9], [10], [5] e [11], ou utilizam técnicas de Software para predição, detecção e isolamento de sensores defeituosos [12] e [13], ou mesmo recorrem à criptografia para tolerar falhas maliciosas que podem afetar os sistemas $e$-Health em tempo real [14].

No aspecto de tolerância a falhas, neste artigo, apresentamos uma abordagem complementar aos trabalhos relacionados, que consiste em um detector de defeitos ao nível de hardware. O 
detector proposto previne que ECGs com sinais de sensores defeituosos, que extrapolam certas faixas de operação, ou estejam desconectados da pele, sejam analisados, alertando o operador da máquina para a realização de nova aquisição ou ajuste da operação. Embora a utilização de sensores redundantes, com custo adicional de hardware, viabilize o mascaramento sem a necessidade de detecção de defeitos, mesmo nestes casos, a detecção pode ser usada para indicar a substituição ou ajuste do sensor, mantendo o nível de confiabilidade do sistema. Além disso, para certos tipos de defeitos detectáveis ao nível do hardware, nosso detector evita análises mais custosas e desnecessárias ao nível do software. As técnicas acima descritas - redundância de sensores, detecção ao nível de software e detecção ao nível de hardware - podem ser utilizadas em conjunto para aumentar a robustez do serviço de ECG por meio digital (e-health).

Neste artigo, apresentamos o projeto, a implementação e avaliação da ferramenta Cyber-ECG, que inclui o detector de defeitos de sensores acima descrito e todos os mecanismos necessários para processamento e visualização de ECGs. Na Cyber-ECG, as séries temporais que representam os ECGs são processadas e submetidos a uma Máquina de Estados Finita (MEF) original, do tipo Mealy, para a tomada de decisões na classificação dos diferentes tipos de ECGs - entre arritmias cardíacas e ECGs normais. Para testar o detector de defeitos, foi implementado um subsistema injetor de falhas de Hardware e um sistema híbrido para a detecção dos defeitos dos sensores. Neste último, foi programado um circuito comparador analógico ativo com histereses, o qual simula a parte de detecção de um eletrocardiógrafo real. Finalmente, foram usados vários parâmetros de avaliação de desempenho que permitiram comparar os resultados da ferramenta CyberECG com outros sistemas correlatos de $e$-Health. A ferramenta Cyber-ECG foi implementada no ambiente Simulink/Matlab. A seguir, é descrita a estrutura deste artigo.

$\mathrm{Na}$ Seção II, é apresentada uma contextualização teórica. $\mathrm{Na}$ Seção III, é descrita a arquitetura da ferramenta Cyber-ECG. Já na Seção IV, é apresentada a metodologia de implementação utilizada, dando ênfase às principais contribuições. Na Seção $\mathrm{V}$, é realizada uma avaliação da ferramenta Cyber-ECG. Por fim, na Seção VI, são apresentados os resultados e as perspectivas futuras.

\section{ContextualizaçÃo TeóricA}

\section{A. Natureza bioelétrica do coração}

O sistema cardiovascular é composto por três tipos principais de tecidos musculares: o músculo atrial, os ventrículos e as fibras especializadas. O coração é constituído por quatro compartimentos: dois átrios (direito e esquerdo) e dois ventrículos (direito e esquerdo). A parede do coração é denominada miocárdio. Os fenômenos bioelétricos cardíacos são causados pelos fluxos dos íons através das membranas celulares das células do coração, chamadas miócitos [15]. Cada ciclo ou batimento cardíaco é acompanhado por dois processos básicos, denominados Sístole e Diástole. O primeiro consiste em um período de contração ventricular, onde o coração expele o sangue dos ventrículos ao resto do corpo e aos pulmões. Na Diástole ocorre um processo de relaxamento ventricular, durante o qual os ventrículos se enchem de sangue, provenientes dos átrios.

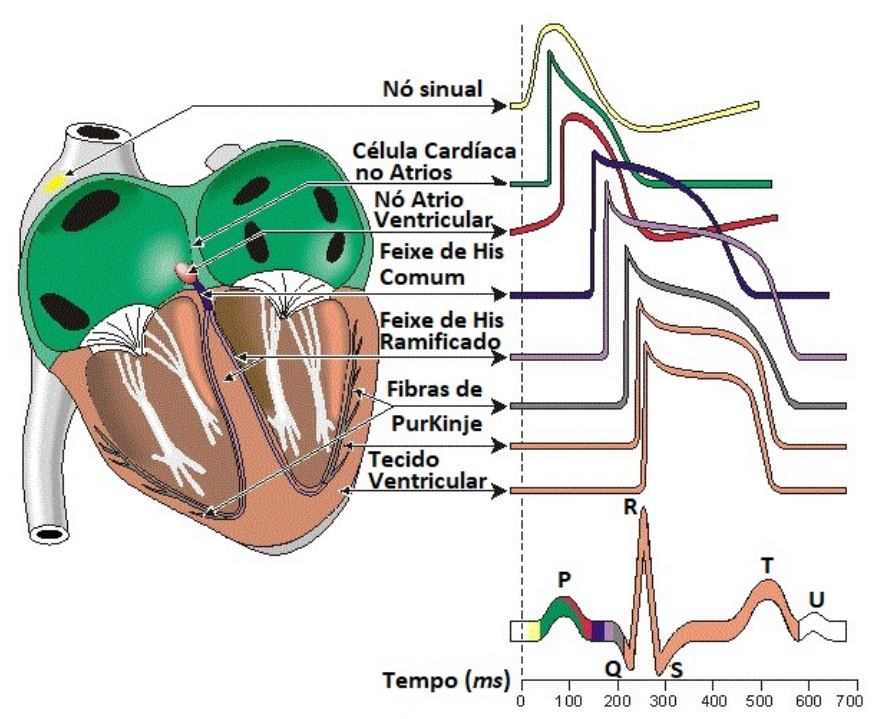

Figura 1. Corte longitudinal do coração visualizando as suas principais partes. $\mathrm{Na}$ parte superior podemos observar os átrios e na inferior os ventrículos. Além disso, estão ilustradas as diferentes regiões de ativação do potencial de ação cardíaca, origem da formação do eletrocardiograma. Figura adaptada de [15].

Na Figura 1, mostramos o corte longitudinal do coração e os locais de ativação elétrica cardíaca, onde se originam os diferentes potenciais de ação cardíacos. Todos estes potenciais contribuem para a formação dos ECGs em uma pessoa. Desta forma, podemos afirmar que um sinal de ECG é constituído por diferentes segmentos, ou ondas, chamadas $\mathrm{P}, \mathrm{Q}, \mathrm{R}, \mathrm{S}, \mathrm{T}$ e $\mathrm{U}$, para cada ciclo cardíaco. Estas ondas servem de referência para definir intervalos temporais (segmentos - ST) e conjuntos de ondas (complexos - QRS), cujos valores constituem um dos critérios de avaliação para a extração dos diferentes estados de saúde do miocárdio [16]. Por exemplo, ECGs com ritmos cardíacos anormais, chamados arritmias, ocorrem devido às alterações na sequência normal de impulsos elétricos do coração. Estas arritmias podem ser originadas tanto nos átrios quanto nos ventrículos. Quando as arritmias ocorrem nos ventrículos, existe uma alta probabilidade de acontecer um infarto do miocárdio, colocando em risco a vida do paciente [2].

\section{B. Classificação de Arritmias em Tempo Real}

Em condições normais, medir a frequência cardíaca é muito simples, por exemplo, com aparelhos monitores que são muito acessíveis atualmente. No entanto, este método de aferir não substitui de forma alguma os exames para realizar diagnósticos precisos de arritmias [17].

A detecção de arritmias é realizada por algoritmos que analisam os sinais de ECGs em tempo real, a partir da extração dos parâmetros dos complexos QRS válidos. Um exemplo destes 
parâmetros é o intervalo de tempo entre os complexos QRS, o qual é tradicionalmente usado para calcular a frequência cardíaca, e assim classificar o tipo de sinal cardíaco [2]. Dentre dos métodos de processamento digital destes sinais, se destacam aqueles baseados na teoria de Redes Neurais, nas Transformadas de Hilbert ou de Fourier, nos Modelos de Markov, nos Algoritmos Genéticos, na Matemática Morfológica, nas Transformadas Wavelet contínua ou discretas, no algoritmo de Pan-Tompkins, dentre outros [18], [19], [20], [21] e [22].

Neste trabalho, aplicamos o algoritmo de Pan-Tompkins, o qual detalharemos adiante. A escolha deste algoritmo de processamento dos sinais de ECGs se deu pela ampla utilização e aceitação deste método. Entretanto, independente do método para classificação de ECGs, quando estes são associados às aplicações de e-Health, são caracterizados como sistemas críticos, pois as falhas podem interferir na configuração dos ECGs analisados e resultar em diagnósticos imprecisos. Ou seja, qualquer outro método de classificação poderia ser utilizado em nossa ferramenta em conjunto com os demais componentes da Cyber-ECG.

\section{Fundamentos de Tolerância a Falhas}

A confiabilidade (reliability) é um atributo da dependabilidade (dependability), definido como a probabilidade de um determinado sistema desempenhar suas funções projetadas, conforme certas condições de operação, em um dado intervalo de tempo [23]. O principal meio para obtenção de sistemas mais confiáveis é a Tolerância a Falhas. Nesta abordagem, parte-se da premissa de que falhas são inevitáveis, portanto algum mecanismo deve ser incorporado ao sistema para tratar as falhas que eventualmente possam ocorrer. Os termos falhas (faults), erros (errors) e defeitos (failures), seguem o padrão da terminologia adotada internacionalmente na área de dependabilidade [23]. A especificação precisa de quais falhas podem ocorrer em um sistema é denominada Modelo de Falhas do sistema [24]. Para o cenário de e-Health com base na Eletrocardiografia, as falhas estão frequentemente relacionadas com a qualidade da conectividade elétrica entre o dispositivo de aquisição (os sensores) e a pele [10] e [5], podendo resultar na omissão ou em erros nos valores captados pelos sensores.

Neste trabalho, propomos a implementação de um detector de defeitos com a finalidade de verificar o funcionamento dos sensores, de modo que, quando estes forem acometidos por falhas, ações possam ser tomadas, tais como: a substituição de componentes defeituosos, recuperação ou reconfiguração do sistema ou uma parada segura [24]. Para facilitar o teste de funcionamento do detector, criamos um procedimento de inserção artificial de falha, como será visto adiante.

\section{Visão Geral da FerRamenta Cyber-ECG}

A ferramenta computacional Cyber-ECG é constituída dos seguintes módulos principais: Aquisição de ECGs (simulando o Eletrocardiógrafo); Processamento Digital dos ECGs; Modelo de Decisões para classificação de ECGs; e, por último, a Visualização dos Resultados. A sua dinâmica de funcionamento é mostrada no fluxograma da Figura 2.

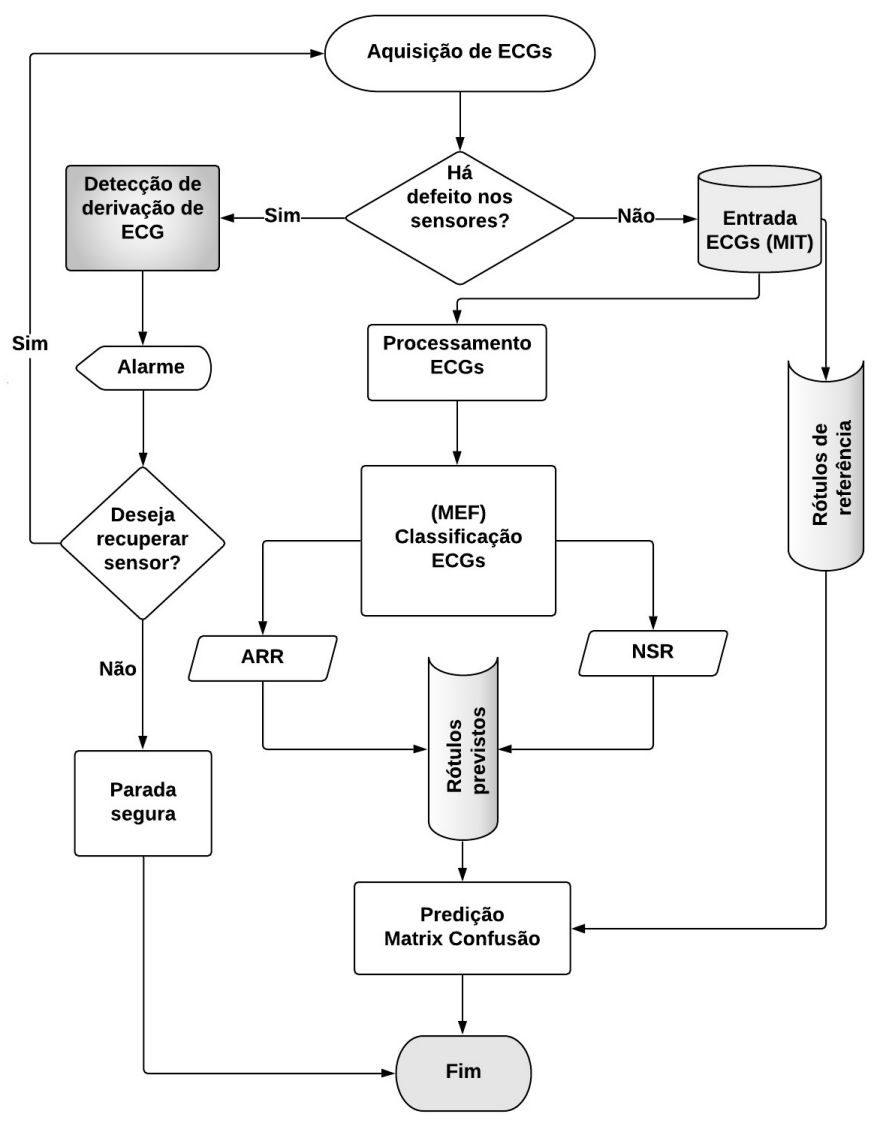

Figura 2. Fluxograma do funcionamento da ferramenta Cyber-ECG.

A ferramenta inicia sua execução através do módulo de aquisição dos ECGs, ativando o bloco de detecção de defeitos que permite verificar se há falhas nas conexões dos sensores (derivações cardíacas). Se há defeito na conexão entre sensores e a pele, $L E D s$ indicativos de defeitos dos sensores ficarão vermelhos na interface gráfica do usuário. Uma vez o defeito detectado, o sistema realiza uma parada segura das análises ou a reinicialização da ferramenta.

Se não há defeito nos sensores, então os $L E D s$ indicadores de defeitos dos sensores ficarão verdes e, em seguida, é executada a etapa de aquisição dos ECGs. A aquisição em nosso sistema é feita através da leitura de séries temporais que representam ECGs reais. Estas séries temporais são enviadas para o estágio de processamento, usando o bloco ECGin Goto do Simulink, e são recebidas pelo bloco ECGin From no subsistema de detecção das derivações de ECGs. Posteriormente, é realizado o processamento digital dos sinais de ECGs, obtendo-se a frequência cardíaca, usada como variável de entrada na MEF, onde classificamos os sinais de ECGs nas categorias NSR (normal) ou ARR (arritmia). Os resultados são então apresentados na interface gráfica do usuário, como será mostrado adiante a partir de tela da ferramenta Cyber-ECG. 


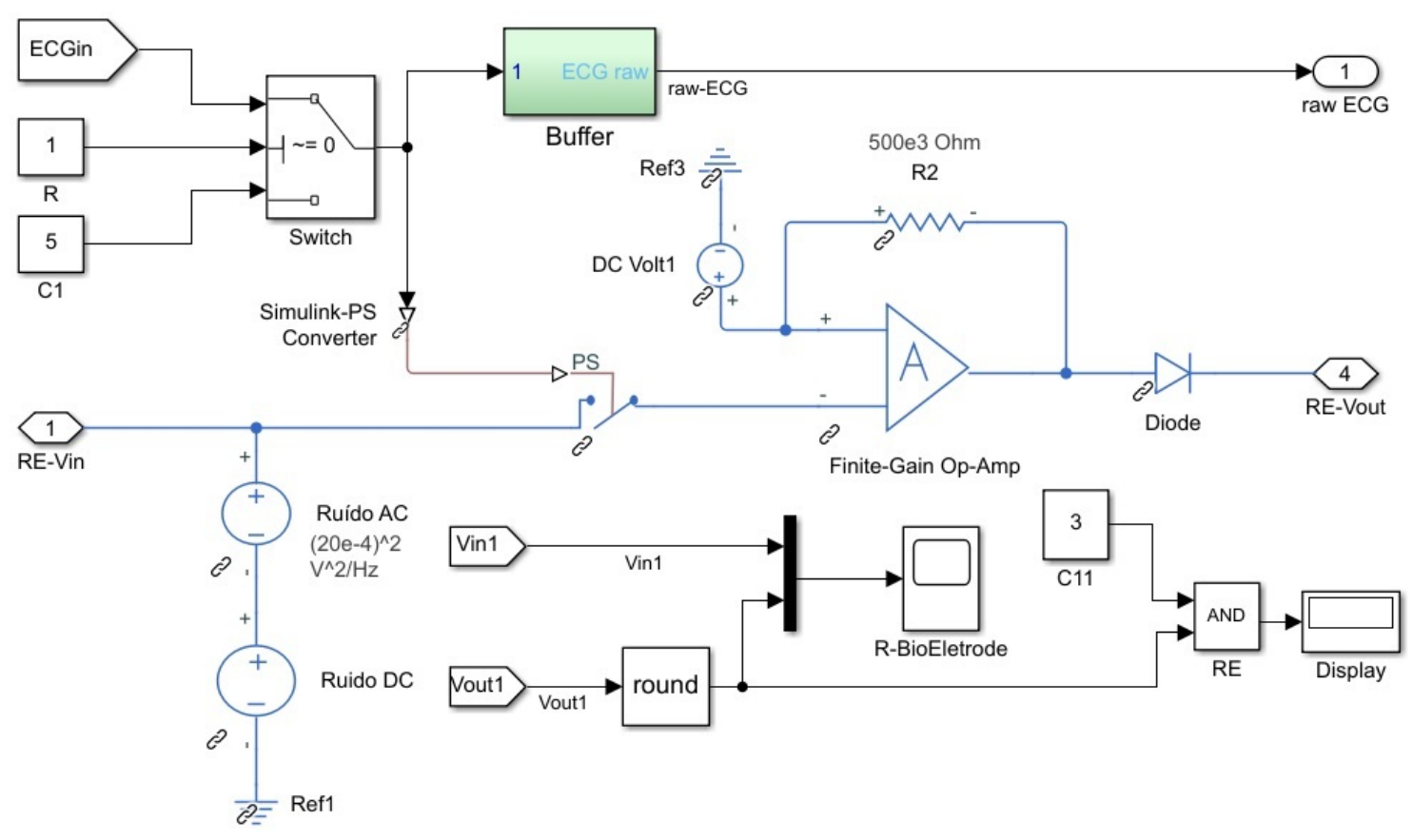

Figura 3. Circuito para a detecção de defeitos de Hardware, usando um comparador analógico com histerese.

\section{Detalhamento da ImplementaçÃo da FERRAMENTA CYBER-ECG}

A seguir descrevemos em mais detalhes cada um dos módulos da ferramenta Cyber-ECG e como são visualizados no ambiente Matlab/Simulink.

\section{A. Aquisição de ECGs}

O bloco de entrada de ECGs é visualizado por um fantoma digital humano, que ilustra o sistema de aquisição de ECGs. A configuração é composta por três sensores: LA (braço esquerdo); RA (braço direito); e sensor RL (perna direita). Este último é chamado sensor de referência, usado para reduzir os ruídos dos cabos de conexão.

\section{B. Detector de Defeitos}

Este bloco detecta defeitos na continuidade elétrica entre os sensores de ECG e a pele, monitorando o nível de impedância. Ou seja, conforme a conectividade do sensor é interrompida, a impedância em série aumenta, sendo tal aumento a indicação de uma falha. Na Figura 3, ilustramos o detector de defeitos, que simula a detecção DC nas derivações cardíacas. Este circuito analógico pode ser implementado em um eletrocardiógrafo real. Na ferramenta Cyber-ECG, usamos três detectores de defeitos, um para cada sensor ou derivação cardíaca.

O componente principal do detector de defeitos é um amplificador operacional, configurado como um comparador, composto por uma configuração de histerese, através de um resistor de $500 \mathrm{k} \Omega$. Além disso, o circuito possui um elevado ganho de $10^{5}$ e uma alimentação de $5 \mathrm{~V}$, garantindo assim um funcionamento mais estável. A saída do comparador foi conectada a um diodo para retificar a voltagem elétrica medida.
E na entrada não inversora do amplificador, foi fixado um valor de tensão igual a $2.5 \mathrm{~V}$ e, na inversora, um sinal DC de amplitude $1 \mathrm{~V}$, ao qual the foi adicionado um sinal $\mathrm{AC}$ de ruído branco, que simula a desconexão ou mau posicionamento dos sensores com a pele (descrita na Seção V).

Caso os sensores estejam bem configurados, ou seja, nenhum defeito seja detectado, os interruptores $(R, L$ e $N)$ enviam sinais lógicos de alto nível para a porta de entrada $(R)$ da chave lógica digital Switch. Neste caso, a chave fica no estado lógico verdadeiro e os sinais de ECGs na primeira porta de entrada ECGin passam para o estágio de processamento, através de um Buffer. Nesta situação, para o interruptor PS no circuito comparador ficar aberto, os sinais bioelétricos cardíacos devem permanecer no limite de impedância, estabelecido no comparador histerese. Na parte inferior da Figura 5, apresentada mais à frente, podemos ver a saída amplificador com valor da tensão medida de $(0)$ volts, que indica a atuação do detector em cada sensor, em tempo real.

\section{Processamento dos sinais de ECGs}

Caso nenhum defeito seja detectado, é realizado o processamento e/ou filtragem dos sinais de ECGs. Para isto, foi aplicado um filtro passa-banda FIRI de ordem 256 e uma banda de passagem de 5 a $26 \mathrm{~Hz}$, com o intuito de eliminar sinais de ruídos de diferentes fontes. Em seguida, é obtida a derivada do sinal filtrado, através de um filtro derivativo de ordem 51, visando eliminar os ruídos provenientes da atividade muscular vizinha ao coração. É calculado, então, o valor absoluto para cada sinal de ECG e determinada a média dos valores absolutos, com um filtro mediador com uma janela de $80 \mathrm{~ms}$. Posteriormente, os sinais cardíacos são processados pelo bloco de detecção $Q R S$, que detecta os picos 


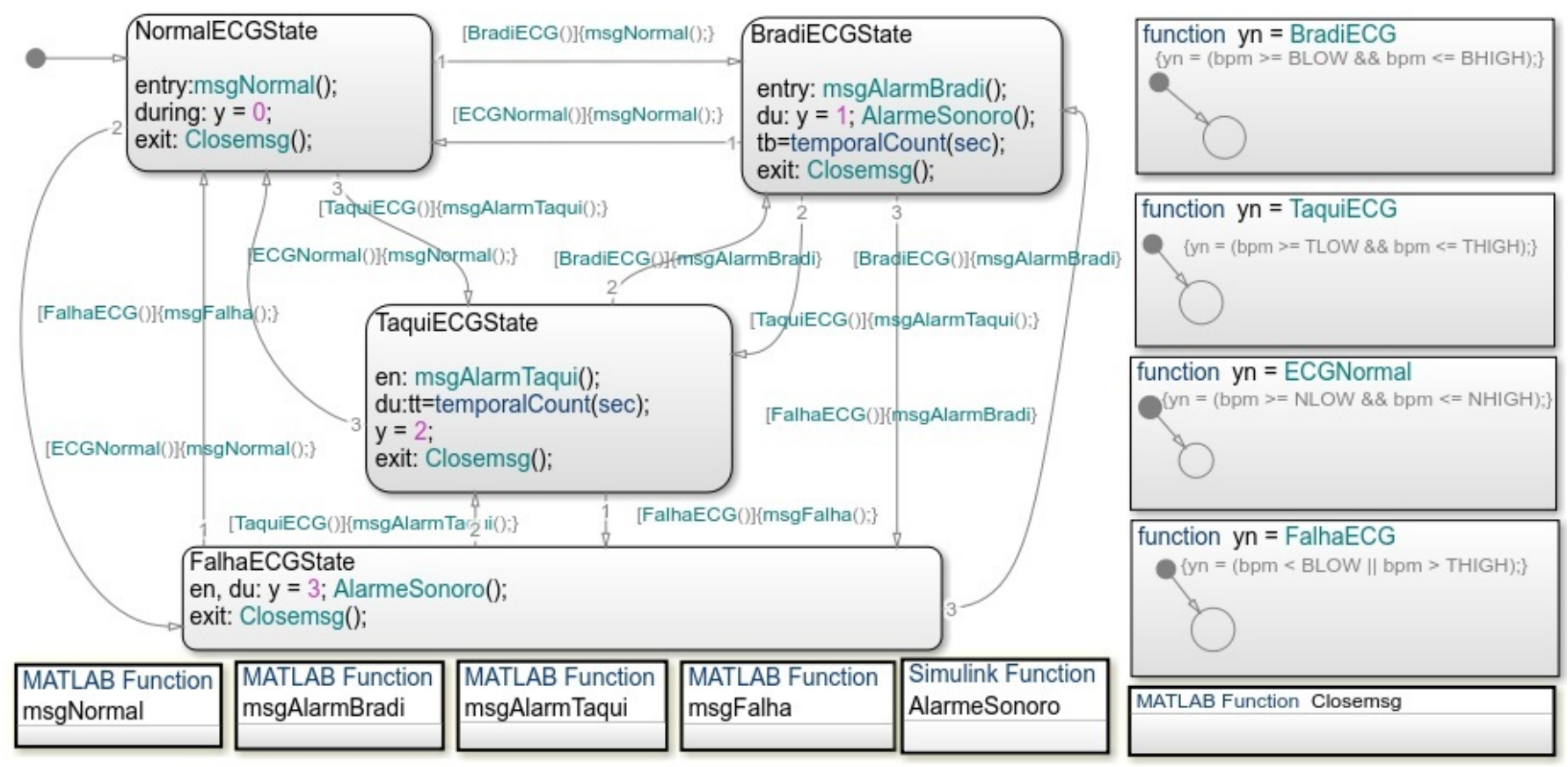

Figura 4. Visão Simulink/Matlab da Máquina de Estados Finita utilizada para a classificação de arritmias cardíacas na Cyber-ECG.

QRS dos sinais de ECGs filtrados em tempo real, e cria uma série temporal com os picos RR. O método Pan-Tompkins é utilizado para extrair a taxa de batimentos cardíacos $(T B C)$, dada em batimentos por minutos (bpm). Um complexo $Q R S$ é dito normal quando a duração for inferior a $120 \mathrm{~ms}$ [25]. Para calcular a $T B C$, foi necessário determinar o valor médio do intervalo de tempo entres os picos $R R(t R R)$. Seguindo a regra apresentada por [26], o valor do intervalo $t R R$ é válido se for menor que $196 \mathrm{~ms}$. Este último tempo corresponde a uma $T B C$ de 306 bpm, maior que a frequência cardíaca máxima teórica de um ser humano que é de $300 \mathrm{bpm}$ [27]. Considerando o $t R R$ em $m s$, a $T B C$ em bpm foi calculada usando a seguinte equação 1:

$$
T B C=\frac{1000 \times 60}{t R R}
$$

\section{Classificação de ECGs}

A classificação de ECGs é feita através de um autômato ou Máquina de Estados Finitos (MEF) do tipo Mealy, a qual foi programada usando uma lógica de decisão sequencial da biblioteca Stateflow ${ }^{\circledR}$ do Simulink. Na Figura 4, aparecem os principais componentes da MEF, com quatro estados excludentes: (NormalECGState, BradiECGState, TaquiECGState e FalhaECGState). Também fazem parte da MEF: quatro funções Matlab (msgNormal, msgAlarmBradi, msgAlarmTaqui, msgFalha); uma função Simulink chamada AlarmeSonoro; e quatro funções gráficas da biblioteca Stateflow (msgNormal, msgAlaemBradi, msgAlarmTaqui e msgFalha). O estado inicial é normal (NormalECGState). Os demais estados são classificados e transitam segundo as faixas de valores da $T B C$, conforme a Tabela I [25]. Como será visto adiante, a interface gráfica do usuário permite a visualização dos estados com alarmes sonoros.

Tabela I

CLASSIFICAÇÃO DE ARRITMIAS CARDÍACAS, DEPENDENDO DOS VALORES standards DA TAXA DE BATIMENTOS CARDÍACOS (TBC), DE UM SUJEITO ACORDADO E EM REPOUSO, CONFORME AS DIRETRIZES DA AAC PARA O MANEJO DE PACIENTES COM ARRITMIAS VENTRICULARES E A PREVENÇÃO DA MORTE CARDÍACA SÚBITA [28].

\begin{tabular}{|c|c|c|c|}
\hline \hline $\begin{array}{c}\text { Estado } \\
\text { do Miocárdio }\end{array}$ & Parâmetros & Valores & Condições Lógicas \\
\hline BradiECG & BLOW & 49 & bpm $>=$ BLOW \&\& \\
& BHIGH & 59 & bpm $<=$ BHIGH \\
\hline ECGNormal & NLOW & 60 & bpm $>=$ NLOW \&\& \\
& NHIGH & 100 & bpm $<=$ NHIGH \\
\hline TaquiECG & TLOW & 101 & bpm $>=$ TLOW \&\& \\
& THIGH & 250 & bpm $<=$ THIGH \\
\hline Disfunção Crítica & - & - & bpm $<$ BLOW \|l \\
& & & bpm $>$ THIGH \\
\hline \hline
\end{tabular}

Os parâmetros mostrados na Tabela I seguem os critérios da Associação Americana do Coração (AAC) [28], os quais especificam que o coração está funcionando normalmente em uma pessoa adulta, sem problemas cardíacos e em estado de repouso, se sua frequência cardíaca oscila no intervalo de 60 a 100 bpm. Quando o coração não está batendo em um padrão regular, se caracteriza a arritmia cardíaca - as mais comuns são a bradicardia e a taquicardia [28].

Além destes parâmetros, a MEF também utiliza as diretrizes de [25] para ativar os estados TaquiECGState e BradiECGState. Estas diretrizes estabelecem que uma taquicardia polimórfica persistente é identificada quando são detectados 3 ou mais complexos QRS consecutivos originados nos ventrículos, com a $T B C$ maior que $100 \mathrm{bpm}$ e menor que $250 \mathrm{bpm}$, durante 

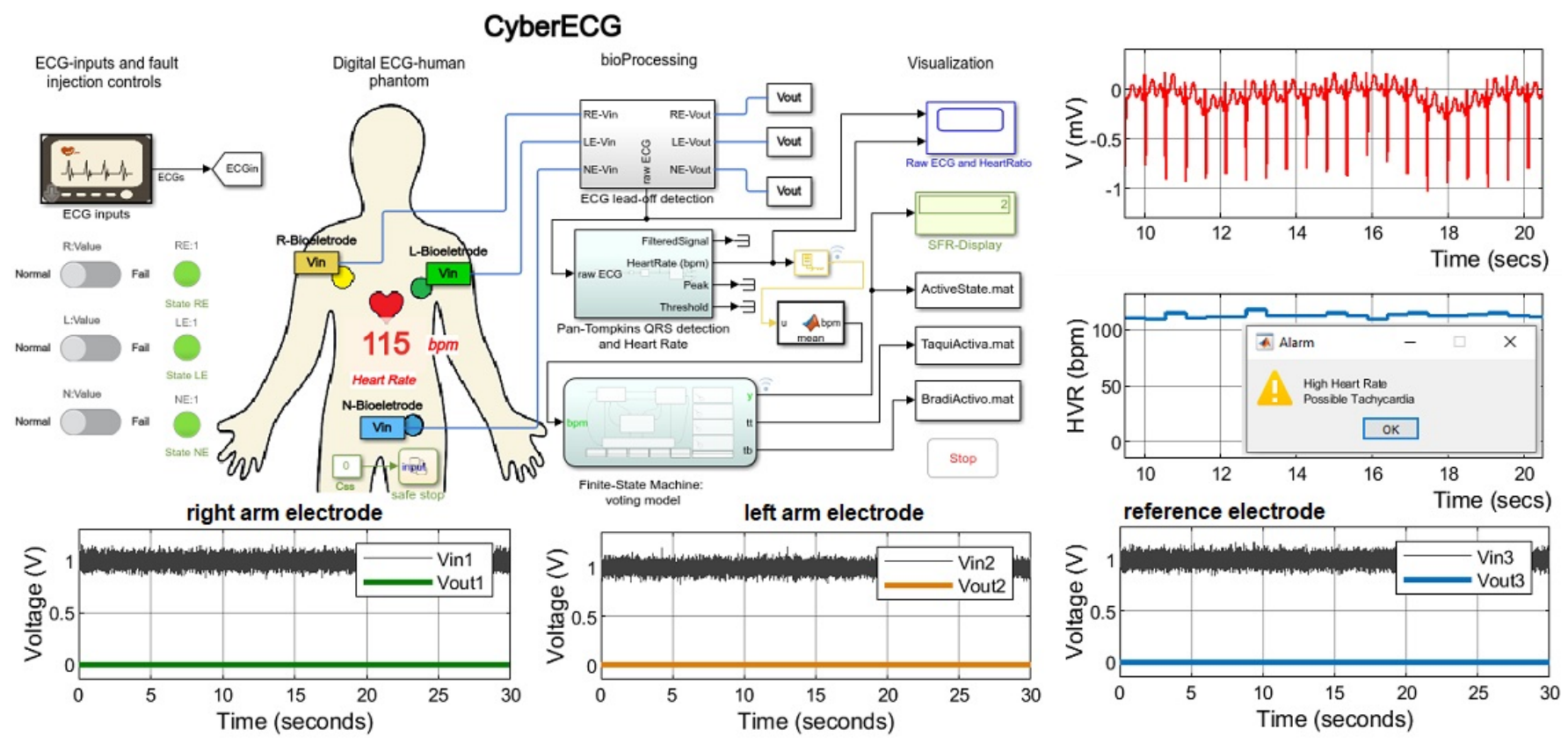

Figura 5. Interface gráfica de usuário da ferramenta computacional Cyber-ECG, funcionando sem injeção de falhas.

um intervalo de tempo maior ou igual que $30 \mathrm{~s}$. E de forma similar, a detecção de uma bradicardia persistente, caso sejam detectados 3 ou mais complexos QRS consecutivos, com uma $T B C$ menor que $60 \mathrm{bpm}$ e maior que $49 \mathrm{bpm}$ durante um tempo maior ou igual que $30 \mathrm{~s}$. Portanto, dependendo do valor da $T B C$ e das diretrizes expressas acima, o Cyber-ECG classifica o ECGs com arritmia (ARR) ou normal (NSR).

\section{E. Visualização dos Resultados}

O bloco de visualização dos resultados consiste na identificação por diferentes avisos visuais e sonoros, tanto para o paciente em observação, quanto para o profissional clínico (ver Figura 5). No entanto, é importante salientar que caberá ao médico responsável tomar as condutas médicas necessárias para resguardar a saúde do paciente, conforme as informações disponíveis nos alarmes.

\section{AVAliando o Cyber-ECG usando ECGs Reais}

Para verificar o funcionamento do Cyber-ECG foi utilizado o banco de dados MIT-BIH que contém registros de sinais de ECGs ambulatoriais, obtidos de 47 indivíduos estudados pelo Laboratório de Arritmia BIH entre 1975 e 1979, com 25 homens com idades entre 32 e 89 anos e 22 mulheres com idades entre 23 e 89 anos [29]. Para os testes foram utilizadas 20 ECGs com arritmias MIT-BIH-AR (ARR) [30] e 20 ECGs com ritmo sinusal normal MIT-BIH-N (NSR) [31]. Estes sinais cumprem as recomendações da Associação para o Avanço da Instrumentação Médica (AAMI) [32]. Inicialmente, todos os ECGs extraídos da base de dados passaram por um préprocessamento. Este constitui na re-amostragem para o valor de $128 \mathrm{~Hz}$, com uma resolução de conversão analógica/digital de 11 bits, e uma faixa da amplitude de até $10 \mathrm{mV}$. Também cada série temporal dos ECGs ficou com uma duração total de $512 \mathrm{~s}$.

Na Figura 5, é mostrada a ferramenta Cyber-ECG funcionando sem defeito dos sensores, ou seja, com os LEDs indicativos na cor verde. Na tela em questão, foi selecionado um sinal de ECG da categoria ARR. Neste instante, foram identificadas as formas de ondas e sua $T B C$ correspondente. Neste teste, a MEF ativou de forma correta o estado TaquiECGState, resultando em valores da variável de saída $y=2$ - também é possível verificar o seu resultado da classificação através do aviso na janela de mensagens. O mesmo procedimento foi realizado para outros sinais de ECGs em estados distintos, com a identificação correta dos variáveis $y=0$ ou $y=1 \mathrm{e}$, também das suas respectivas mensagens relacionadas.

Para testar o detector de defeito foi implementado um subsistema de injeção de falhas. Este mecanismo foi baseado em exemplo apresentado por [33], através do qual o usuário pode induzir a condição de falha e depois repará-la. Os testes realizados permitiram simular falhas no dispositivo medidor de ECGs ou a inoperância dos sensores. Nesta situação, uma falha simulada significa a liberação de sinal $\mathrm{AC}$ de ruído branco, que resulta em uma tensão fixa de $5 \mathrm{~V}$ na saída do comparador. Portanto, caso a tensão dos sensores não estejam nos limites estabelecidos, ou seja, maior que 0 e menor que $2,5 \mathrm{~V}$, o interruptor PS é fechado, impedindo o processamento do ECG.

$\mathrm{Na}$ hipótese do injetor de falha seja ativado, o usuário identifica a inoperância dos sensores a partir dos LEDs da entrada, que mudarão para vermelho e a variável recebida na porta de entrada $R$, da chave Switch, muda para y $=3$, ativando o estado FalhaECGState. Em seguida, é acionada a função AlarmeSonoro, sendo indicada a realização de uma 
parada segura do sistema, com ativação do botão Stop e, assim, permitindo a entrega de uma análise correta e o reparo dos sensores.

\section{A. Métricas de Desempenho}

Para calcular o desempenho do Cyber-ECG na classificação de ECGs foram usados vários parâmetros ou métricas de avaliação. Os parâmetros são: a Sensibilidade (Sen) ou Recall, Especificidade (Esp), Valor Preditivo Positivo ou Precisão (PPV), Acurácia(Acc) e Score (F1), que podem ser obtidas a partir das seguintes equações 2 - 6 :

$$
\begin{gathered}
\text { Sen }=\operatorname{Recall}(\%)=\frac{V P}{V P+F N} \times 100 \% \\
\operatorname{Esp}(\%)=\frac{V N}{V N+F P} \times 100 \% \\
P P V=\operatorname{Precis} \tilde{a} o(\%)=\frac{V N}{V N+F P} \times 100 \% \\
\text { Acc }(\%)=\frac{V P+V N}{V P+V N+F P+F N} \times 100 \% \\
F 1-\operatorname{Score}(\%)=\frac{2 V P}{2 T P+F P+F N} \times 100 \%
\end{gathered}
$$

As expressões anteriores foram definidas em função das variáveis verdadeiros positivos $(\mathrm{VP})^{1}$, verdadeiros negativos $(\mathrm{VN})^{2}$, falsos positivos $(\mathrm{FP})^{3}$, e falsos negativos $(\mathrm{FN})^{4}$.

\section{B. Avaliação de Desempenho}

Para avaliar os resultados, foi utilizado o método de análise preditiva ou classificação estatística na área da inteligência computacional [17]. Neste campo, no que diz respeito à avaliação de modelos, é comumente utilizada a matriz de confusão. Em nosso caso foi obtida uma matriz de confusão de tamanho $2 \times 2$, conforme as classes verdadeiras e previstas, como podem ser vistos na Figura 6.

Para obtenção das métricas de desempenho de forma automática foi usada uma rotina escrita em Matlab, adaptada de [34]. Desta forma, os ECGs selecionados para análise são primeiramente randomizados, com auxílio de uma da matriz de tamanho de [40x65536] (chamada PredictedLabelsECG.mat) e, após os resultados inferidos pela ferramenta Cyber-ECG serem gravados, seus rótulos de classificação são comparados com os rótulos da matriz de referência (Reference.mat). Estes últimos são fornecidos pela base de dados MIT-BIH e representam classificações validadas por especialistas em saúde. Portanto, a partir desta comparação são obtidos os valores VP, VN, FP e FN, usados para calcular as equações $2-6$ e gerar a matriz de confusão.

\footnotetext{
${ }^{1}$ VP: quantidade de sinais de ECGs que o Cyber-ECG identificou como arritmias

${ }^{2}$ VN: ECGs que o Cyber-ECG identificou como normal corretamente.

${ }^{3}$ FP: ECGs do tipo NSR, que o Cyber-ECG classifica incorretamente como de ARR.

${ }^{4} \mathrm{FN}$ : ECGs da classe ARR, preditos pelo Cyber-ECG incorretamente na classe NSR
}
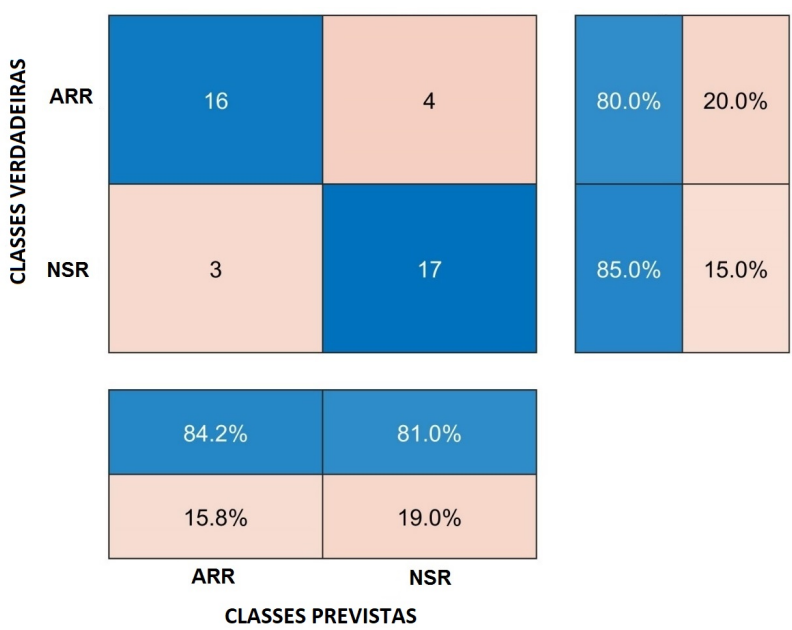

Figura 6. Matriz de confusão estendida para o modelo Cyber-ECG, para as classes ou categorias ARR e NSR.

Na mesma Figura 6, são reportados 16 e 17 ECGs classificados corretamente, sendo 4 e 3 os classificados incorretamente, para as classes ARR e NSR, respectivamente. Desta forma, foi obtida uma acurácia de $82.5 \%$, pois classificou corretamente 33 sinais de ECGs, nas suas respectivas categorias. Também apresentou uma sensibilidade ou recall de $80 \%$ em prever sinais de ECGs na classe ARR e NSR. E realizou esta classificação com uma precisão de $84.2 \%$.

O Cyber-ECG, a partir de adaptações ao algoritmo de PanTompkins, apresentou um desempenho comparável com os trabalhos realizados por [35], [36] e [37], que reportaram uma precisão de $80 \%, 81,2 \%$ e $84.3 \%$, respectivamente. Em comparação com os estudos reportados por [38], [39], os quais apresentam precisões de $60 \%$ e $76.42 \%$ respectivamente, o Cyber-ECG apresentou desempenho consideravelmente melhor. Acreditamos que este desempenho foi possível devido à versatilidade da MEF em se adaptar às mudanças das diversas categorias de sinais de ECGs.

\section{CONCLUSÕES}

As tecnologias computacionais de e-health apresentam grandes desafios tecnológicos e científicos, em particular na garantia de confiabilidade desses sistemas. Neste contexto, apresentamos o projeto e implementação da ferramenta computacional Cyber-ECG para a classificação automática de sinais de ECGs. O Cyber-ECG inclui um detector de defeito dos sensores no nível do hardware e uma MEF original do tipo Mealy para a classificação automática dos sinais de ECGs. Para avaliação da Cyber-ECG no escopo da classificação de ECGs, foram randomizados 40 ECGs reais (20 ARR e 20 NSR), a partir do banco de dados público MIT-BIH, observandose o bom desempenho na classificação realizada, quando comparada a trabalhos similares. Para teste do detector de defeito, criamos um subsistema de injeção de falhas e deteç̧ão de defeitos, simulando um eletrocardiógrafo real.

Em trabalhos futuros pretendemos estudar a confiabilidade do detector de defeitos em ambientes físicos reais, coletando 
sinais de ECGs em tempo real. Além disso, estudaremos a integração de outros mecanismos de tolerância a falhas, como a redundância de sensores e predição de defeitos por análise do comportamento temporal dos sensores. Também planejamos adicionar novos estados à MEF, tais como pressão arterial e temperatura, ampliando assim o potencial de possíveis aplicações médicas para o Cyber-ECG.

\section{REFERÊNCIAS}

[1] W. H. O. WHO et al., "Who reveals leading causes of death and disability worldwide: 2000-2019," Retrieved February, vol. 21, p. 2021, 2020.

[2] S. B. SBC et al., "Cardiômetro: Mortes por doênças cardiovasculares no brasil, 2016," Dados dos anos anteriores. [acessado em 04/08/2016]. Disponivel em http://www. cardiometro. com. br/anteriores. asp, 2017.

[3] M. U. Jakobsen, E. Trolle, M. Outzen, H. Mejborn, M. G. Grønberg, C. B. Lyndgaard, A. Stockmarr, S. K. Venø, and A. Bysted, "Intake of dairy products and associations with major atherosclerotic cardiovascular diseases: a systematic review and meta-analysis of cohort studies," Scientific reports, vol. 11, no. 1, pp. 1-28, 2021.

[4] G. Eysenbach, "What is e-health?," Journal of medical Internet research, vol. 3, no. 2, p. e20, 2001.

[5] F. Abdali-Mohammadi, V. Bajalan, and A. Fathi, "Toward a fault tolerant architecture for vital medical-based wearable computing," Journal of medical systems, vol. 39, no. 12, p. 149, 2015.

[6] S. R. Islam, D. Kwak, M. H. Kabir, M. Hossain, and K.-S. Kwak, "The internet of things for health care: a comprehensive survey," IEEE Access, vol. 3, pp. 678-708, 2015.

[7] R. Horst, D. Jewett, and D. Lenoski, "The risk of data corruption in microprocessor-based systems," in Fault-Tolerant Computing, 1993. FTCS-23. Digest of Papers., the Twenty-Third International Symposium on, pp. 576-585, IEEE, 1993.

[8] M. Schmidt and R. Obermaisser, "Adaptive and technology-independent architecture for fault-tolerant distributed aal solutions," Computers in biology and medicine, 2017.

[9] R. Sharifi and R. Langari, "Sensor fault diagnosis with a probabilistic decision process," Mechanical systems and signal processing, vol. 34, no. 1-2, pp. 146-155, 2013.

[10] T. N. Gia, A.-M. Rahmani, T. Westerlund, P. Liljeberg, and H. Tenhunen, "Fault tolerant and scalable iot-based architecture for health monitoring," in 2015 IEEE Sensors Applications Symposium (SAS), pp. 1-6, IEEE, 2015.

[11] C. C. Oliveira and J. M. Da Silva, "Fault diagnosis in highly dependable medical wearable systems," Journal of Electronic Testing, vol. 32, no. 4, pp. 467-479, 2016.

[12] D.-J. Kim and B. Prabhakaran, "Motion fault detection and isolation in body sensor networks," Pervasive and Mobile Computing, vol. 7, no. 6 , pp. 727-745, 2011.

[13] M. Merah, A. Ouamri, A. Nait-Ali, and M. Keche, "Fault tolerant neural network for ecg signal classification systems," Advances in Electrical and Computer Engineering, vol. 11, no. 3, pp. 17-24, 2011.

[14] S. A. Kumar, B. Bhargava, R. Macêdo, and G. Mani, "Securing iot-based cyber-physical human systems against collaborative attacks," in Internet of Things (ICIOT), 2017 IEEE International Congress on, pp. 9-16, IEEE, 2017.

[15] J. Malmivuo and R. Plonsey, Bioelectromagnetism: principles and applications of bioelectric and biomagnetic fields. Oxford University Press, USA, 1995.

[16] D. De Araujo, A. A. Carneiro, E. R. Moraes, and O. Baffa, "Biomagnetismo: nova interface entre a fisica ea biologia," Ciência Hoje, pp. 24-29, 1999.

[17] C. Chen, Z. Hua, R. Zhang, G. Liu, and W. Wen, "Automated arrhythmia classification based on a combination network of cnn and 1stm," Biomedical Signal Processing and Control, vol. 57, p. 101819, 2020.

[18] V. Krasteva, I. Jekova, R. Leber, R. Schmid, and R. Abächerli, "Realtime arrhythmia detection with supplementary ecg quality and pulse wave monitoring for the reduction of false alarms in icus," Physiological measurement, vol. 37, no. 8, p. 1273, 2016.

[19] B. Vandendriessche, M. Abas, T. E. Dick, K. A. Loparo, and F. J. Jacono, "A framework for patient state tracking by classifying multiscalar physiologic waveform features," IEEE Transactions on Biomedical Engineering, vol. 64, no. 12, pp. 2890-2900, 2017.
[20] Q. Li, C. Rajagopalan, and G. D. Clifford, "Ventricular fibrillation and tachycardia classification using a machine learning approach," IEEE Transactions on Biomedical Engineering, vol. 61, no. 6, pp. 1607-1613, 2013.

[21] Z. I. Attia, S. Kapa, F. Lopez-Jimenez, P. M. McKie, D. J. Ladewig, G. Satam, P. A. Pellikka, M. Enriquez-Sarano, P. A. Noseworthy, T. M Munger, et al., "Screening for cardiac contractile dysfunction using an artificial intelligence-enabled electrocardiogram," Nature medicine, vol. 25 , no. 1 , p. $70,2019$.

[22] J. Pan and W. J. Tompkins, "A real-time qrs detection algorithm," IEEE transactions on biomedical engineering, no. 3, pp. 230-236, 1985.

[23] A. Avizienis, J.-C. Laprie, B. Randell, and C. Landwehr, "Basic concepts and taxonomy of dependable and secure computing," IEEE transactions on dependable and secure computing, vol. 1, no. 1, pp. 11-33, 2004

[24] R. J. A. Macêdo and J. A. Farines, "Projeto de sistemas distribuídos e de tempo real para automação. edufba, 2018, 250p," 2018.

[25] C. Pastore, C. Pinho, H. Germiniani, N. Samesima, and R. Mano, "Diretrizes da sociedade brasileira de cardiologia sobre análise e emissão de laudos eletrocardiográficos," Arquivos Brasileiros de Cardiologia, vol. 93, no. 3, pp. 1-19, 2009.

[26] P. S. Hamilton, "Open source ecg analysis software documentation," Computers in cardiology, vol. 2002, pp. 101-104, 2002.

[27] L. Chhabra, N. Goel, L. Prajapat, D. H. Spodick, and S. Goyal, "Mouse heart rate in a human: diagnostic mystery of an extreme tachyarrhythmia," Indian pacing and electrophysiology journal, vol. 12, no. 1, pp. 32-35, 2012.

[28] S. M. Al-Khatib, W. G. Stevenson, M. J. Ackerman, W. J. Bryant, D. J. Callans, A. B. Curtis, B. J. Deal, T. Dickfeld, M. E. Field, G. C. Fonarow, A. M. Gillis, C. B. Granger, S. C. Hammill, M. A. Hlatky, J. A. Joglar, G. N. Kay, D. D. Matlock, R. J. Myerburg, and R. L. Page, "2017 aha/acc/hrs guideline for management of patients with ventricular arrhythmias and the prevention of sudden cardiac death," Circulation, vol. 138, no. 13, pp. e272-e391, 2018.

[29] A. L. Goldberger, L. A. Amaral, L. Glass, J. M. Hausdorff, P. C. Ivanov, R. G. Mark, J. E. Mietus, G. B. Moody, C.-K. Peng, and H. E. Stanley, "Physiobank, physiotoolkit, and physionet: components of a new research resource for complex physiologic signals," circulation, vol. 101, no. 23, pp. e215-e220, 2000.

[30] M. PhysioNet, "Arrhythmia database," URL: https://www. physionet. org/physiobank/database/mitdb, 2017.

[31] A. Goldberger, L. Amaral, L. Glass, J. Hausdorff, P. Ivanov, R. Mark, J. Mietus, G. GB, C. Peng, and H. Stanley, "The mit-bih normal sinus rhythm database," Circulation, vol. 101, no. 23, pp. e215-e220.

[32] AAMI et al., "American national standard for ambulatory electrocardiographs, publication ansi," AAMI EC38-1994, 1994.

[33] E. E. A. Hussein et al., Fault Tolerance Analysis in Fuel Control System Using SIMULINK Software. PhD thesis, Sudan University of Science \& Technology, 2016.

[34] L. T. Patrick E. McSharry, Gari D. Clifford and L. A. Smith, "A dynamical model for generating synthetic electrocardiogram signals," IEEE Transactions On Biomedical Engineering, vol. 50, no. 3, pp. 289294, 2003.

[35] S. Tuli, N. Basumatary, S. S. Gill, M. Kahani, R. C. Arya, G. S. Wander, and R. Buyya, "Healthfog: An ensemble deep learning based smart healthcare system for automatic diagnosis of heart diseases in integrated iot and fog computing environments," Future Generation Computer Systems, vol. 104, pp. 187-200, 2020.

[36] Q. Yao, R. Wang, X. Fan, J. Liu, and Y. Li, "Multi-class arrhythmia detection from 12-lead varied-length ecg using attention-based timeincremental convolutional neural network," Information Fusion, vol. 53, pp. 174-182, 2020.

[37] J. Chu, H. Wang, and W. Lu, "A novel two-lead arrhythmia classification system based on cnn and 1stm," Journal of Mechanics in Medicine and Biology, vol. 19, no. 03, p. 1950004, 2019.

[38] V. K. Varadana, P. Raib, S. C. Ohb, and P. S. Kumarb, "Wearable technology and mobile platform for human health monitoring," in Forum Electromagn. Res. Methods Appl. Technol, vol. 16, pp. 1-38, 2016.

[39] T. M. Inc., "Signal classification using waveletbased features and support vector machines," URL:https://la.mathworks.com/help/wavelet/examples/ecgclassification-using-wavelet-features.html, 2020. 